\title{
Low serum albumin levels predict poor outcome in patients with acute ischaemic stroke or transient ischaemic attack
}

To cite: Zhou H, Wang A, Meng $X$, et al. Low serum albumin levels predict poor outcome in patients with acute ischaemic stroke or transient ischaemic attack. Stroke \& Vascular Neurology 2021;0. doi:10.1136/svn-2020-000676

- Additional material is published online only. To view, please visit the journal online (http://dx.doi.org/10.1136/svn2020-000676).

$\mathrm{HZ}$ and $\mathrm{AW}$ contributed equally.

Received 10 0ctober 2020 Revised 6 January 2021 Accepted 24 January 2021

\section{Check for updates}

(c) Author(s) (or their employer(s)) 2021. Re-use permitted under CC BY-NC. No commercial re-use. See rights and permissions. Published by BMJ.

${ }^{1}$ Department of Neurology, Beijing Tiantan Hospital, Capital Medical University, Beijing,

China

${ }^{2}$ China National Clinical

Research Center for

Neurological Diseases, Beijing

Tiantan Hospital, Capital Medical

University, Beijing, China

${ }^{3}$ Tiantan Neuroimaging Center of Excellence, Beijing Tiantan Hospital, Capital Medical University, Beijing, China ${ }^{4}$ Advanced Innovation Center for Human Brain Protection, Capital Medical University, Beijing, China

Correspondence to Dr Yongjun Wang; yongjunwang@ncrcnd.org.cn

\section{ABSTRACT}

Background To examine the relationship of serum albumin with poor functional outcome and mortality in patients with acute ischaemic stroke (AIS) or transient ischaemic attack (TIA), and perform a meta-analysis to summarise the association.

Methods We analysed data from the Third China National Stroke Registry (CNSR-III). Patients were divided into four groups based on serum albumin levels at admission. The outcomes included poor functional outcome (modified Rankin Scale (mRS) score of 3 to 6) and mortality at 3 months and 1 year. Multiple logistic regression models and Cox regression models were used to evaluate the association, respectively. We used a fixed-effect model to calculate the risk ratio for poor functional outcome and a random-effect model for mortality in the meta-analysis. Results A total of 13618 patients were enrolled. During the 3-month follow-up period, compared with 40 to $44.9 \mathrm{~g} / \mathrm{L}$ group, patients in $<35 \mathrm{~g} / \mathrm{L}$ group had an increased risk of poor functional outcome and mortality (adjusted OR 1.37 (95\% Cl 1.12 to 1.67); adjusted HR 2.13 (95\% Cl 1.41 to 3.23)). The relationship in per $10 \mathrm{~g} / \mathrm{L}$ decreased serum albumin with prognosis was consistently inversed (adjusted OR 1.17 (95\% Cl 1.01 to 1.35); adjusted HR 1.86 (95\% $\mathrm{Cl} 1.30$ to 2.64)). Also, low serum albumin levels were independently correlated with clinical outcomes at 1 year. In the meta-analysis, the OR for poor functional outcome pooled 3 studies per $1 \mathrm{~g} / \mathrm{L}$ decrease was $1.03(95 \% \mathrm{Cl}$ 1.02 to 1.05$)$, and the HR for mortality pooled 5 studies was 1.07 (95\% Cl 1.03 to 1.11$)$.

Conclusions Low serum albumin levels predict poor functional outcome and mortality in patients with AIS or TIA.

\section{INTRODUCTION}

Albumin is the most abundant serum protein synthesised primarily in the liver and regulated by multiple physiological mechanisms. ${ }^{1}$ It has several physiological properties, including maintaining colloidal osmotic pressure, antiplatelet aggregation and antiinflammatory effects. ${ }^{3}$

Recent pre-clinical research found that moderate-dose albumin therapy after vascular recanalisation remarkably reduced infarction volume and brain swelling, and improved neurological function, suggesting that serum albumin has a neuroprotective effect. ${ }^{4} 5$ However, the results of the Albumin in Acute Stroke (ALIAS) trials on intravenous albumin in patients with stroke were negative disappointedly. ${ }^{6}$ This apparent paradox between the strong basic evidence and the failure in translation to humans brings great confusion. In the past, several studies have discussed the relationship between serum albumin and stroke prognosis, while the sample size was limited and lack long-term follow-up. ${ }^{7-11}$

Our study aimed to assess the correlation between low serum albumin level and poor functional outcome and mortality in patients with acute ischaemic stroke (AIS) or transient ischaemic attack (TIA) in a large, long-term follow-up, prospective registry. Furthermore, we performed a meta-analysis to summarise the association.

\section{METHODS}

\section{Study design and participants}

The Third China National Stroke Registry (CNSR-III) was an ongoing, nationwide, prospective stroke registry enrolling patients with AIS or TIA ( $\geq 18$ years old; within 7 days from the onset) between August 2015 and March 2018. A total of 201 hospitals from 22 provinces and 4 municipalities in China participated. We collected aetiology, imaging and biology data, and tried to clarify the pathogenesis and prognostic factors of ischaemic cerebrovascular disease. Details on the design of the CNSR-III have been published previously. ${ }^{12}$ The study protocol was approved by the ethics committee of all participating study centres. Each participant signed informed consent before enrolment, and was followed up at 3 months and 1 year. An independent 
contract research organisation performed data monitoring throughout the study period.

\section{Data collection}

Baseline information was recorded on admission by trained research coordinators. Some information, like patient demographics, medical history, primary diagnosis, acute recanalisation therapy, inpatient medication and laboratory tests, were extracted from medical records. Other parts were obtained from face-to-face interviews, such as the pre-stroke modified Rankin Scale (mRS) and National Institutes of Health Stroke Scale (NIHSS) scores. The aetiological classification was conducted by the Trial of Org 10172 in Acute Stroke Treatment (TOAST) criteria. ${ }^{13}$ All images were stored in DICOM format on disks and centrally interpreted by two neurologists. Disagreements were resolved by discussion with a third reviewer. The presence of intracranial arterial stenosis and extracranial arterial stenosis was defined as $50 \%-99 \%$ stenosis or occlusion of any intracranial and extracranial artery. ${ }^{1415}$

Serum albumin and alanine aminotransferase (ALT) were tested in sub-centres within the first 24 hours after admission. Bromocresol purple assay or bromocresol green assay were used to measure the serum albumin levels, which depended on the test reagent of sub-centres. High-sensitivity $\mathrm{C}$ reactive protein (hs-CRP), total cholesterol (TC), high-density lipoprotein (HDL), low-density lipoprotein (LDL), triglyceride (TG) and serum creatinine were tested in the central laboratory of Beijing Tiantan Hospital. The blood samples were collected at admission and frozen in the cryotube at $-80^{\circ} \mathrm{C}$ refrigerator, then transported through the cold chain to the central laboratory. The estimated glomerular filtration rate (eGFR) was calculated by Chronic Kidney Disease Epidemiology Collaboration equations. ${ }^{16}$

\section{Outcomes}

The clinical outcomes included functional outcome and mortality, which were obtained through face-to-face interviews at 3 months and 1 year. The poor functional outcome was defined as mRS score of 3 to 6 (disability/ death), and the good functional outcome was defined as mRS score of 0 to 2 (independence). ${ }^{17}$ Mortality included death from all causes.

\section{Statistical analysis}

Continuous variables were presented as mean \pm SD or median with IQR, and categorical variables as percentages. The baseline characteristics of different groups were compared by Kruskal-Wallis test for continuous variables and $\chi^{2}$ test for categorical variables. We used ordinal logistic regression to compare the disparity in mRS scores between different groups at 3 months and 1 year. The proportional hazards assumption of mortality was eligible for Cox regression. The association of serum albumin with poor functional outcome and mortality was analysed in logistic regression models for ORs and
Cox proportional hazards models for HRs, respectively. Moreover, the relationship was tested by two types of data, four groups as categorical variable and every $10 \mathrm{~g} / \mathrm{L}$ as continuous variable. Adjusted ORs, adjusted HRs and their $95 \%$ CI were calculated on three models. In the first model, we adjusted age and sex, which are demographic characteristics. In the second model, we added body mass index (BMI), medical history, index event, TOAST types, pre-stroke mRS score 0 2, NIHSS scores at admission, intracranial arterial stenosis, extracranial arterial stenosis, intravenous thrombolysis and inpatient medication, which were statistically significant in the baseline characteristics comparison. In the third model, we further adjusted the laboratory test, including TG, LDL, HDL, TC, ALT, eGFR and hs-CRP.

Restricted cubic splines were presented as the graphical representation of the associations. The interaction of age, sex, BMI, TOAST types, baseline NIHSS scores and eGFR were analysed by the multivariate logistic model and multivariate Cox model. ${ }^{9} 101819$ Statistical significance was determined as $\mathrm{p}$ values $<0.05$, two-sided. All statistical analyses were performed with SAS software, V.9.4 (SAS Institute, Cary, NG).

\section{Meta-analysis}

The meta-analysis was conducted following the Preferred Reporting in Systematic Reviews and Meta-Analyses statement and the Meta-analysis Of Observational Studies in Epidemiology guidelines (online supplemental table 8). ${ }^{20} 21$ We searched PubMed, Scopus and Embase up to 22 May 2020, using the following combination of search terms: ("albumin" OR "hypoalbuminemia") AND ("stroke" OR "cerebral ischemia" OR "cerebral ischaemia" OR "cerebral infarction" OR "transient ischemic attack" OR "transient ischaemic attack" OR "TIA") AND ("outcome" OR "functional" OR "dependency" OR "mortality" OR "prognosis"). Articles were eligible if (1) the subjects were patients with AIS or TIA, (2) the design was a prospective study, (3) the exposure was serum albumin level (either categorised or continuous), (4) the outcome was poor functional outcome or mortality, and (5) an effect estimate with a 95\% CI was reported. Two reviewers ( $\mathrm{HZ}$ and $\mathrm{AW}$ ) examined the retrieved papers based on the inclusion criteria mentioned previously. Disagreements were resolved by discussion with a third senior reviewer (Y-JW). The most adjusted ORs or HRs were extracted. We used the Newcastle-Ottawa Scale, the funnel plots, Begg's tests and Egger's tests to assess the bias risk of included studies (online supplemental table 7). We performed a fixed-effect model for poor functional outcome and a random-effect model for mortality according to the test of heterogeneity. All analyses were performed using $\mathrm{R}$ software V.3.6.3 (R Foundation for Statistical Computing, Vienna, Austria). More details of statistical analysis are shown in online supplemental material. 


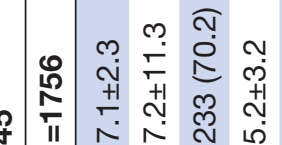

ติ

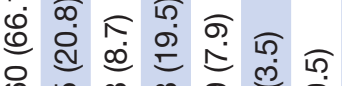

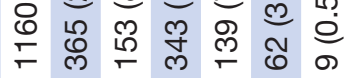

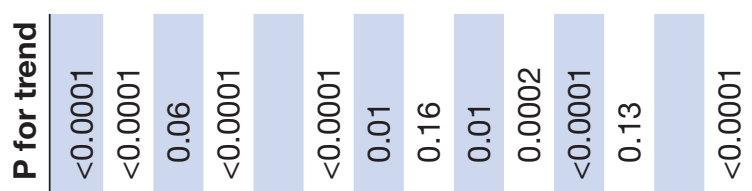

5
웅
v

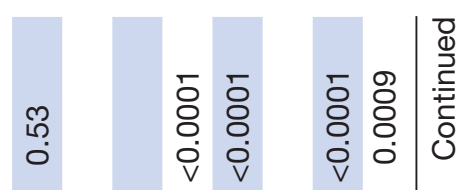

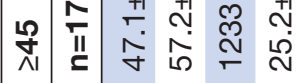

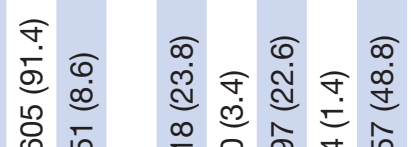

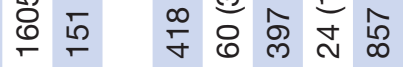

สุ

음

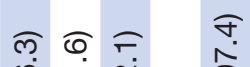

过 这

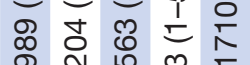

ल

के मे

సิ হั

ल

ลิ ฐิ

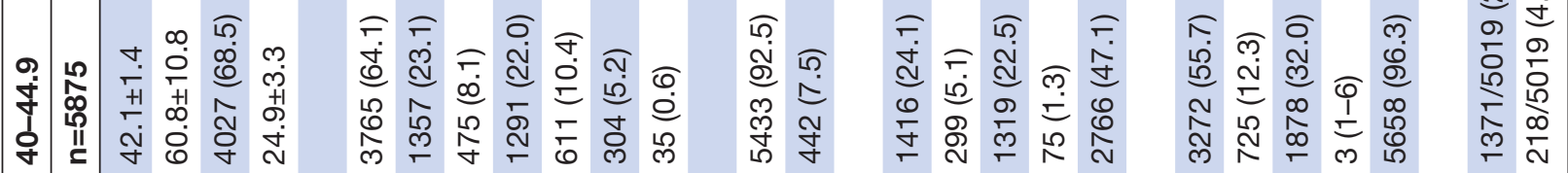

โอ

वे ָิ



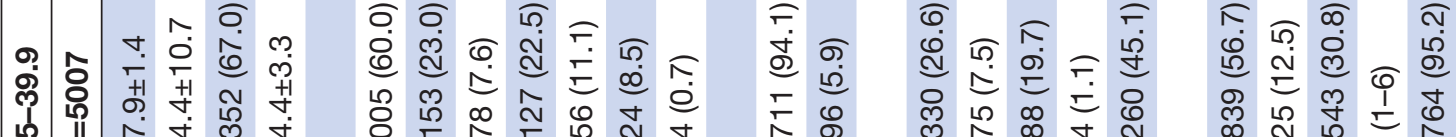
ठे L

岕

产

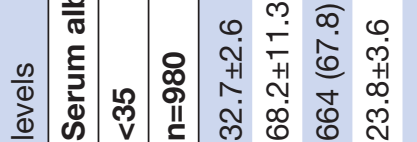

ธุ ธุ

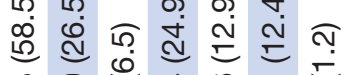

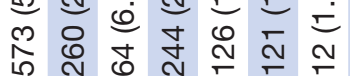

웅

@ )

m.

ชิ จุ $ิ$

ลุ

लि

- i $\quad$ a

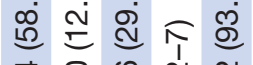

号

సิ

ก

尽

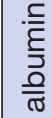

放

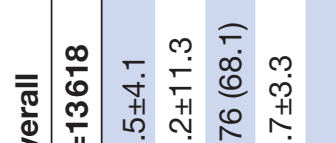

๙ิ

สุ

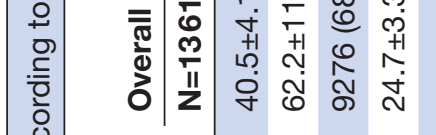

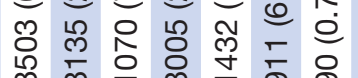

ले $\infty$

लू.

8 0

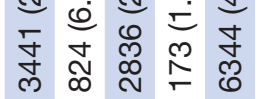

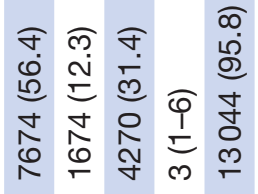

ตุ

$\stackrel{\infty}{\mathfrak{N}} \stackrel{0}{\mathfrak{9}}$

$\stackrel{\text { N }}{\sim}$

\&

$\mp$

సิ స్ స్ల

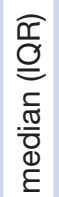

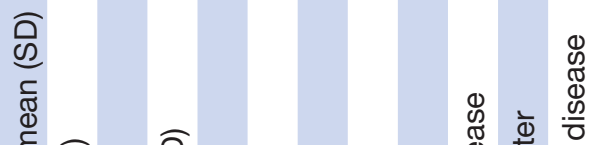

है की बे

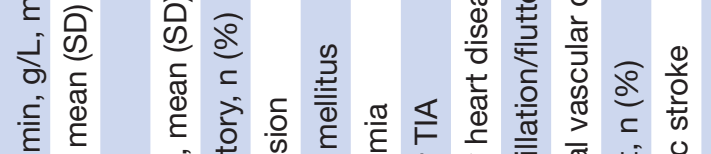

$\frac{\frac{0}{0}}{\frac{\omega}{0}}$

흥 긍

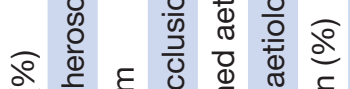

․ำ $\frac{0}{0}$

है के वे

ᄃ

\%

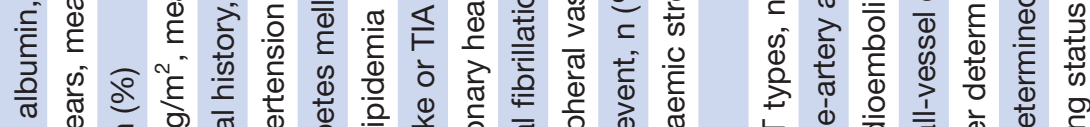

का एँ

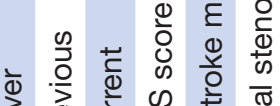

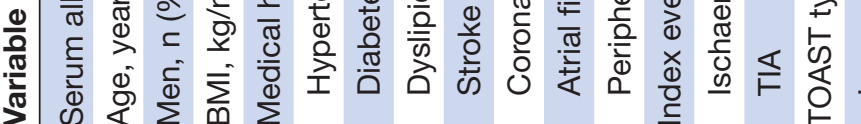

离

है

ये⿺

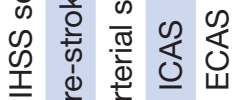

言这走 


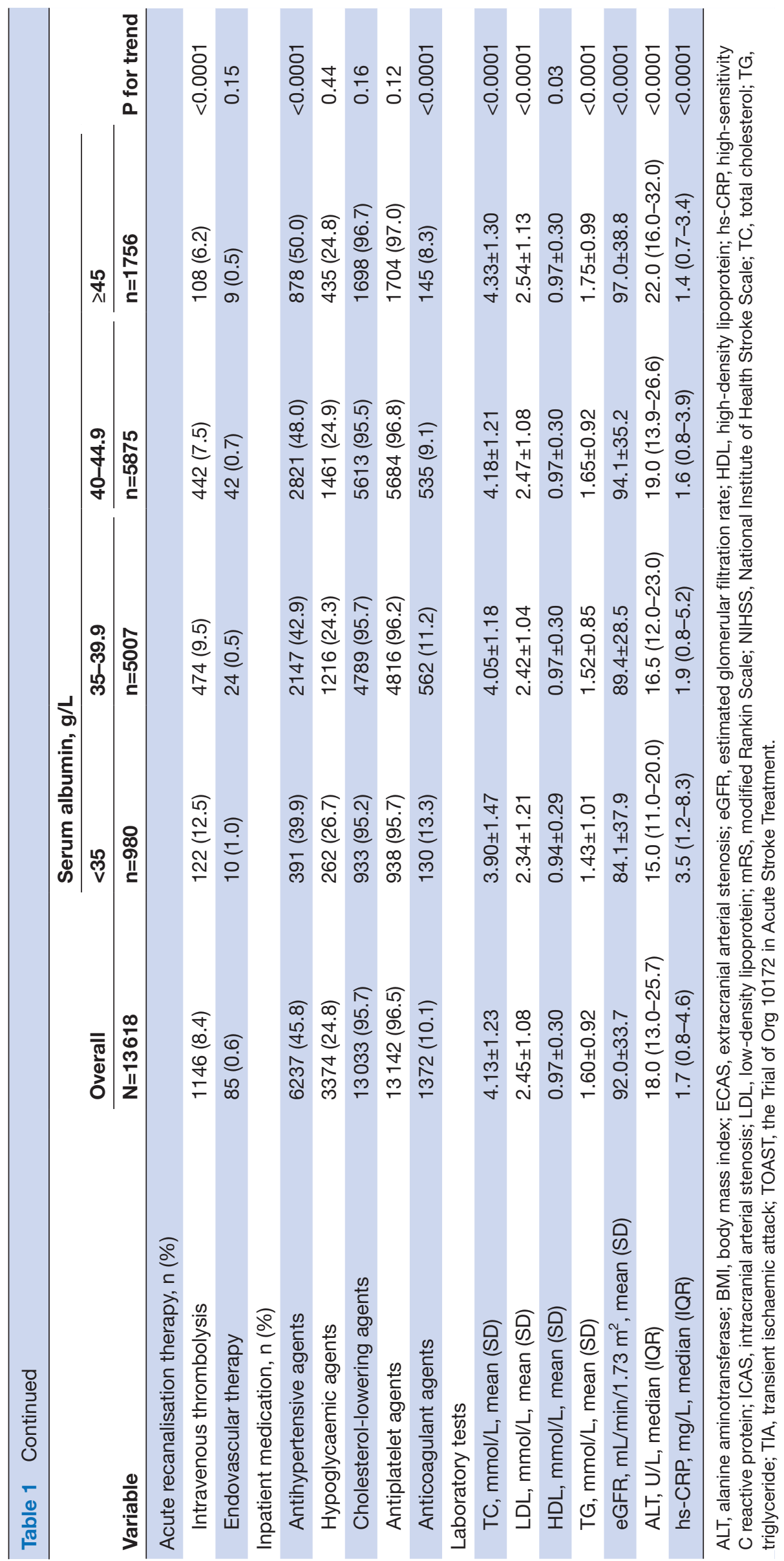


A $\mathrm{mRS}$ at 3 months

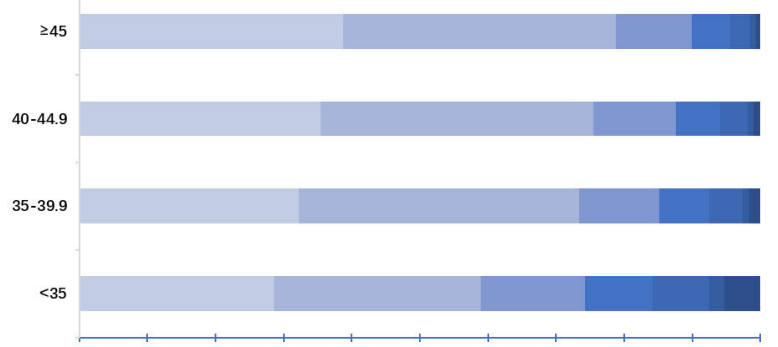

B mRS at 1 year

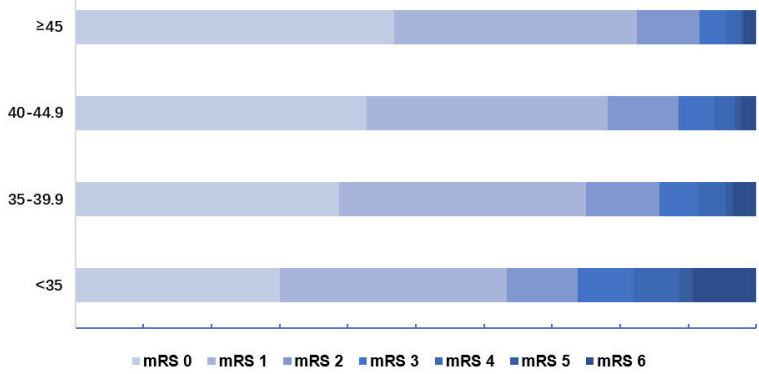

Figure 1 Distribution of $m R S$ score at (A) 3 months and (B) 1 year. mRS, modified Rankin Scale.

\section{RESULTS}

\section{Baseline characteristics}

Among the 15166 patients in the CNSR-III, a total of $13618(89.8 \%)$ participants completed serum albumin measurement and 3-month and 1-year follow-up (online supplemental table 1). They were divided into four groups based on serum albumin levels according to the previous literature: $\quad<35 \mathrm{~g} / \mathrm{L}, \quad 35 \sim 39.9 \mathrm{~g} / \mathrm{L}, \quad 40 \sim 44.9 \mathrm{~g} / \mathrm{L} \quad$ and $\geq 45 \mathrm{~g} / \mathrm{L}$ (online supplemental figure 1). ${ }^{22}$ Overall, 980 $(7.2 \%)$ patients were complicated with hypoproteinemia (defined as serum albumin level $<35 \mathrm{~g} / \mathrm{L}$ ) at admission. ${ }^{23}$ The demographics and clinical characteristics of the study population are shown in table 1 . Compared with the higher serum albumin groups, participants in the lower groups were inclined to be older, had lower BMI, TC, LDL, HDL, TG, eGFR and ALT level, but higher hs-CRP level, higher proportion of large-artery atherosclerosis (LAA) and cardioembolism (CE) in TOAST types, higher proportion of intracranial arterial stenosis and extracranial arterial stenosis, higher percentage of receiving intravenous thrombolysis and anticoagulant agents, and lower percentage of receiving antihypertensive agents. Meanwhile, patients with low serum albumin were more likely to suffer from diabetes mellitus, stroke or TIA, coronary heart disease and atrial fibrillation at baseline, and be dependent before the onset, more severe at admission (table 1).

\section{Clinical outcomes}

As shown in figure 1, the distribution of 3-month and 1-year mRS scores for four groups was significantly different $(p<0.0001)$. During the 3-month follow-up period, $1871(13.7 \%)$ patients had poor functional outcome and 195 (1.4\%) patients died. Disability/died patients had lower serum albumin levels compared with independent patients $(39.6 \pm 4.4 \mathrm{~g} / \mathrm{L}$ vs $40.6 \pm 4.0 \mathrm{~g} / \mathrm{L}$, $\mathrm{p}<0.0001)$. For serum albumin level $<35 \mathrm{~g} / \mathrm{L}$ versus 40 to $44.9 \mathrm{~g} / \mathrm{L}$, crude OR for poor functional outcome was 2.14 (95\% CI 1.81 to 2.52) and crude HR for mortality was 5.03 (95\% CI 3.42 to 7.39). After adjustment for age, sex, BMI, medical history (hypertension, diabetes mellitus, stroke or TIA, coronary heart disease and atrial fibrillation), index event, TOAST types, pre-stroke mRS 0 2, NIHSS scores on admission, intracranial arterial stenosis, extracranial arterial stenosis, intravenous thrombolysis, inpatient medication, serum TG, TC, LDL, HDL, ALT, eGFR and hs-CRP level, the strong association still persisted (adjusted OR 1.37 (95\% CI 1.12 to 1.67); adjusted HR 2.13 (95\% CI 1.41 to 3.23)). In addition, the risk of poor functional outcome and mortality increased $17 \%$ and $86 \%$ for every $10 \mathrm{~g} / \mathrm{L}$ decrease in serum albumin levels (adjusted OR 1.17 (95\% CI 1.01 to 1.35 ); adjusted HR 1.86 (95\% CI 1.30 to 2.64)) (figure 2).

Furthermore, low serum albumin levels also independently predicted poor functional outcome and mortality at 1 year, whether it was a comparison in categorical variables or continuous variables (figure 2 ). As shown in figure 3, restricted cubic spline similarly confirmed the inverse association of decreased serum albumin levels with disability and mortality.

Subgroup analysis was stratified by age, sex, BMI, TOAST types, NIHSS score on admission and baseline eGFR. There were almost no variables that interacted with albumin and clinical outcome, except NIHSS score at admission $\leq 10$ with 3-month poor functional outcome (adjusted OR 1.22 (95\% CI 1.05 to 1.42); p for interaction=0.04) and 3-month mortality (adjusted HR 2.45 (95\% CI 1.56 to 3.85 ); p for interaction=0.02) (online supplemental tables 2 and 3 ).

\section{Meta-analysis}

We identified 4357 relevant articles in initial. There was no meta-analysis of this issue conducted before. After removing 1781 duplicates and excluding 2531 references by title and abstract, 45 articles remained for the second evaluation. Based on the full text, we included six eligible studies in the final (online supplemental figure 2, tables 5 and 6$)$.

There were three studies $(n=14937)$ that reported the association of serum albumin with poor functional outcome. For per $1 \mathrm{~g} / \mathrm{L}$ lower plasma albumin, risk ratio $\left(\mathrm{RR}_{\text {pooled }}\right)$ of poor functional outcome obtained by a fixed-effect model was 1.03 (95\% CI 1.02 to 1.05$)$, without evidence of statistical heterogeneity $\left(\mathrm{I}^{2}=0 \%\right.$; $\mathrm{p}$ for heterogeneity $=0.49$ ) and publication bias based on Begg's tests $(p=0.33)$ and Egger's tests $(p=0.24)$, whereas our research accounted for $80.8 \%$ of the weight (figure 4 ). Five studies $(n=15092)$ were included to explain the relationship between serum albumin and mortality. Due to the significance of statistical heterogeneity $\left(\mathrm{I}^{2}=63 \%\right.$; $\mathrm{p}$ for heterogeneity=0.03), we used a random-effect model to calculate RR ${ }_{\text {pooled }}$ of mortality (1.07 (95\% CI 1.03 to 


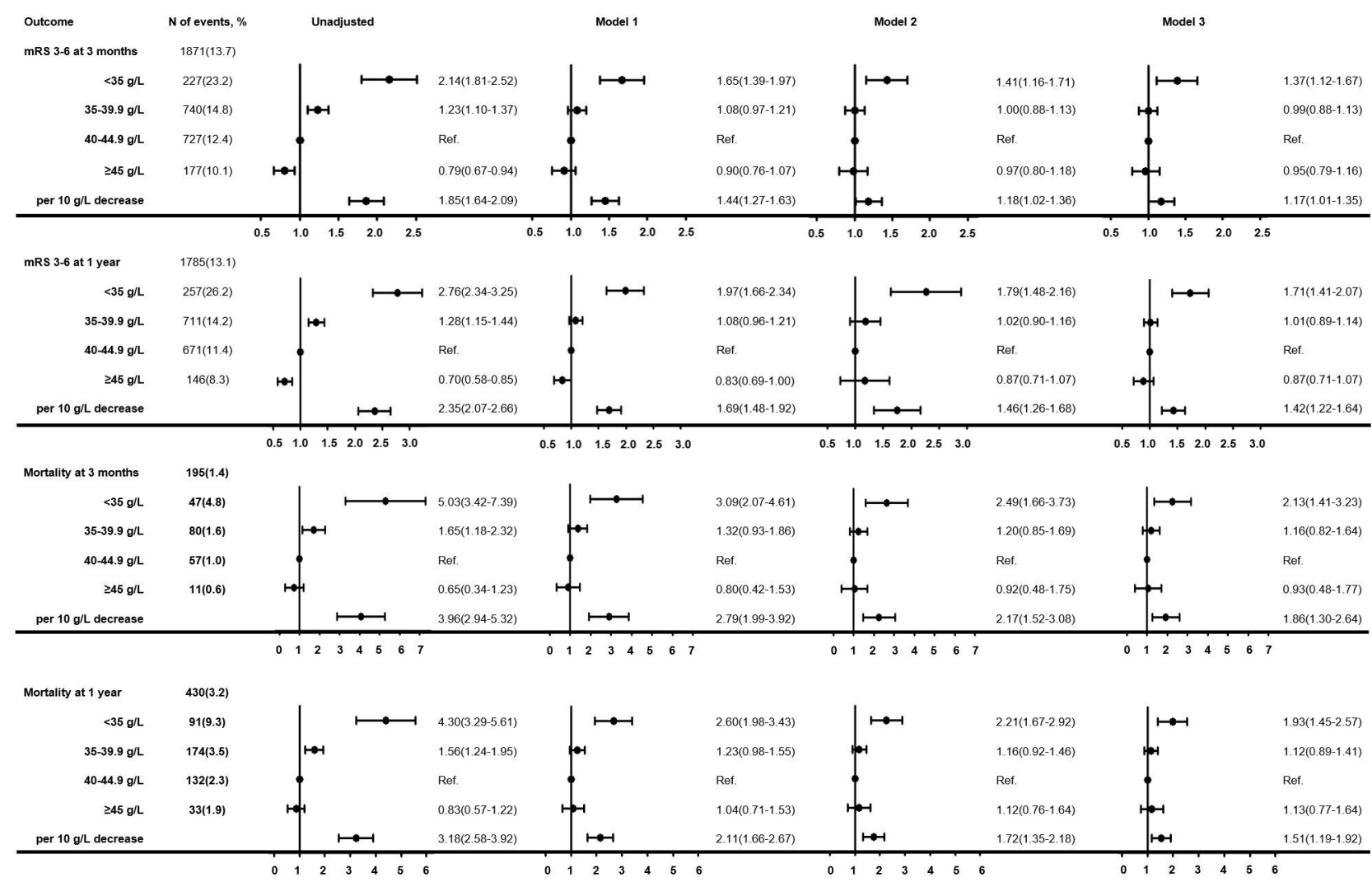

Figure 2 Association of serum albumin with clinical outcomes: HR/OR (95\% Cl). *Model 1: adjusted for age and sex. †Model 2: adjusted for covariables in model 1, plus BMI, medical history (hypertension, diabetes mellitus, stroke or TIA, coronary heart disease and atrial fibrillation/flutter), index event, TOAST types, NIHSS score on admission, pre-stroke mRS 0 2, intracranial arterial stenosis, extracranial arterial stenosis, intravenous thrombolysis and inpatient medication (antihypertensive agents, anticoagulant agents). ¥Model 3: adjusted for covariables in model 2, plus TG, TC, LDL, HDL, ALT, eGFR and CRP at admission. ALT, alanine aminotransferase; BMI, body mass index; eGFR, estimated glomerular filtration rate; HDL, high-density lipoprotein; hs-CRP, high-sensitivity $C$ reactive protein; LDL, low-density lipoprotein; mRS, modified Rankin Scale; NIHSS, National Institute of Health Stroke Scale; TC, total cholesterol; TG, triglyceride; TIA, transient ischaemic attack; TOAST, the Trial of Org 10172 in Acute Stroke Treatment.

1.11)) (figure 4). No publication bias was observed according to Begg's tests $(\mathrm{p}=0.48)$ and Egger's tests $(\mathrm{p}=0.74)$. After excluding Idicula et al, ${ }^{10}$ which had fewer factors to adjusted HR value, there was no statistical heterogeneity $\left(I^{2}=0 \%\right.$; $p$ for heterogeneity $\left.=0.58\right)$, and $R R$ pooled of mortality was still significant (1.05 (95\% CI 1.03 to 1.07)) (online supplemental figure 3 ). The funnel plots are shown in online supplemental figure 4 .

\section{DISCUSSION}

In this prospective study, we confirmed that low serum albumin levels increased the risk of poor functional outcome and mortality in patients with AIS or TIA at 3 months and 1 year, especially in patients accompanying with hypoproteinemia. The results of the meta-analysis were additionally consistent.

In our population, hypoproteinemia accounted for only $7.2 \%$ of patients and varied from the previous data of $33.6 \%$, which may be attributed to improved nutritional status and economic development, as well as the disparity in the inclusion criteria and sample size. ${ }^{7}$ Meanwhile, participants in our research were generally younger than in previous studies. ${ }^{89}$ Since recent publications reported that the incidence of hypoproteinemia in Chinese people over 65 years is $10.1 \%$, and in the white Danish general population is $10.8 \%$, ethnic differences of disease susceptibility cannot be excluded. ${ }^{22}{ }^{24}$ Mild patients were more likely to be affected by albumin. We speculated that patients with severe illness may have more comorbidities or complications, which might mask the role of albumin. ${ }^{9}$

Previous research showed an association between low serum albumin and the incidence of $\mathrm{CE}$ and cryptogenic stroke, but not LAA. ${ }^{18}$ It was well established that low serum albumin levels were linked to many heart diseases, including atrial fibrillation, which was the main cause for $\mathrm{CE},{ }^{25}$ whereas in our study population, the proportion of $\mathrm{CE}$ and LAA both gradually increased with the decrease of serum albumin. Also, the average serum albumin level of patients with CE or LAA was all lower than that of other types (online supplemental table 4). This discrepancy may be partially explained by the different aetiological classification characteristics of patients with stroke in the East and the West. ${ }^{26}$ Even though our research may amplify the effect, there was some relevant evidence supporting 
A

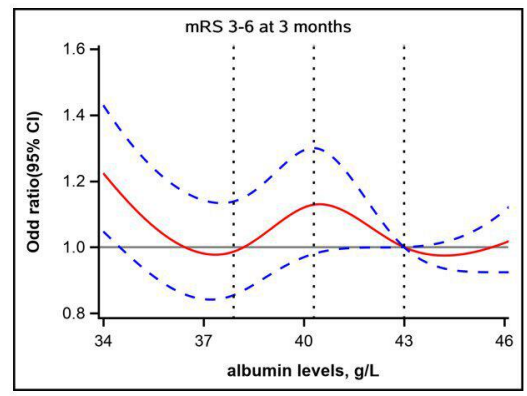

C

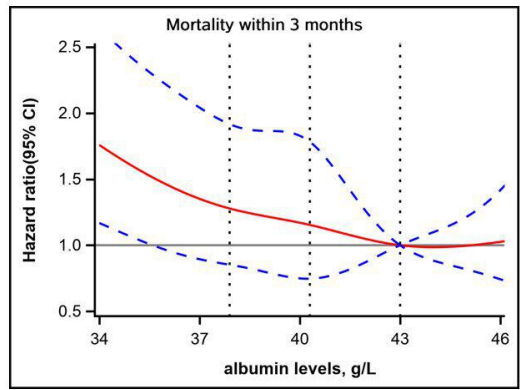

B

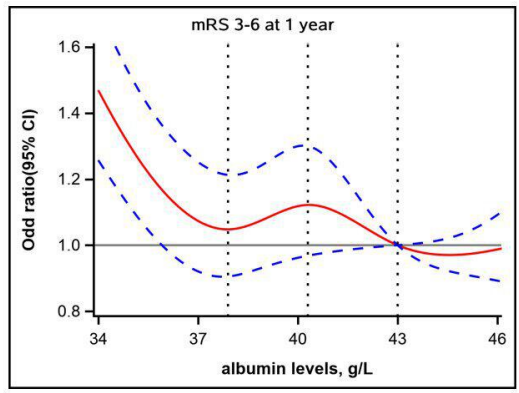

D

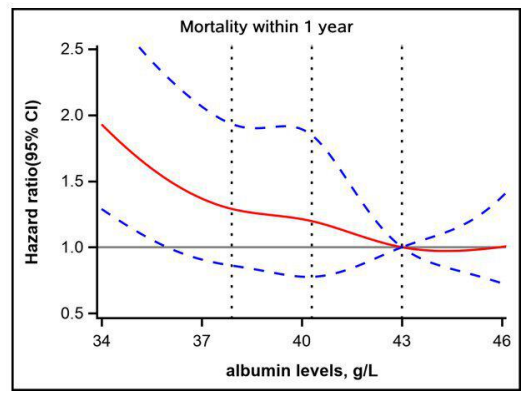

Figure 3 Spline models in the association between serum albumin and clinical outcomes The association with serum albumin level and poor functional outcome (mRS score of 3-6) at (A) 3 months and (B) 1 year. The association with serum albumin level and mortality at (C) 3 months and (D) 1 year. The red line indicates adjusted OR/HR, and the blue lines indicate the $95 \% \mathrm{Cl}$. Data were fitted with a Cox regression model with adjustment for age, sex, BMI, medical history (hypertension, diabetes mellitus, stroke or transient ischaemic attack, coronary heart disease and atrial fibrillation/flutter), index event, TOAST types, pre-stroke mRS score 0 2, NIHSS scores on admission, intracranial arterial stenosis, extracranial arterial stenosis, intravenous thrombolysis, inpatient medication (antihypertensive agents, anticoagulant agents), serum TG, TC, LDL, HDL, ALT, eGFR and hs-CRP. ALT, alanine aminotransferase; BMI, body mass index; eGFR, estimated glomerular filtration rate; HDL, high-density lipoprotein; hs-CRP, high-sensitivity C reactive protein; LDL, low-density lipoprotein; mRS, modified Rankin Scale; NIHSS, National Institute of Health Stroke Scale; TC, total cholesterol; TG, triglyceride; TOAST, the Trial of Org 10172 in Acute Stroke Treatment.

the assumption that serum albumin was associated with LAA. Based on the prior basic medical studies, the weakening anti-inflammatory function, antioxidant effect and endothelial protection of low serum albumin might indirectly promote the development of atherosclerosis. ${ }^{27-29}$

We speculated that the powerful predictive function on poor functional outcome and mortality would be explained by three plausible pathophysiological mechanisms. First, because of a long circulatory half-life, serum albumin might serve as a biomarker of some pre-stroke
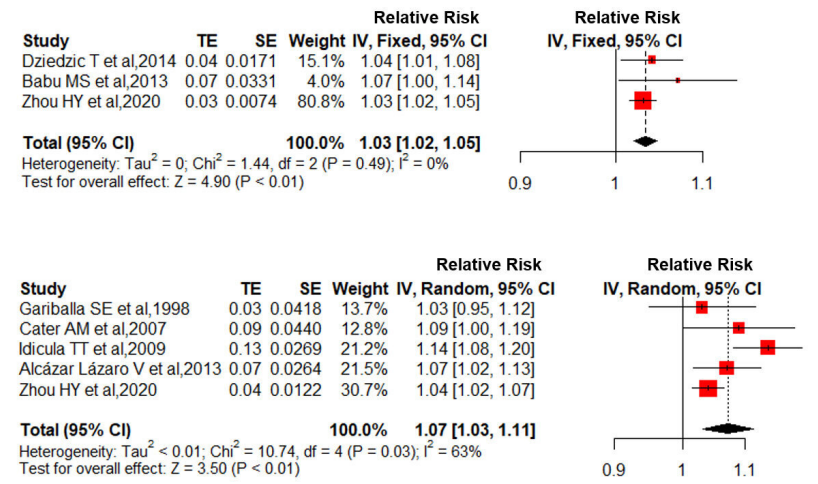

Figure 4 Forest plot of the meta-analysis showed relative risk and $95 \% \mathrm{Cl}$ of $(\mathrm{A})$ poor functional outcome and (B) mortality in every $1 \mathrm{~g} / \mathrm{L}$ decreased serum albumin. TE, treatment effect. pathological states, which seriously affect the prognosis, such as malnutrition. ${ }^{30}$ Nevertheless, we did not find the interaction between BMI and albumin, suggesting that there may be other likely diseases declining serum albumin levels and preventing the rehabilitation, such as kidney disease and cancer. ${ }^{19} 31$ Second, serum albumin deficiency may impair the balance of coagulation and anticoagulation. Early studies have shown that albumin is a cofactor mediating the binding of plasminogen to fibrin, and further participating in the interaction of tissue plasminogen activator. ${ }^{32}$ Also, serum albumin prevented arachidonic acid oxidation and inhibited thromboxane $\mathrm{A}_{2}$ synthesis, which is a potent stimulator of platelet aggregation. ${ }^{33}$ The decrease of serum albumin might weaken the anticoagulant effect and strengthen the coagulation function, and thrombus would be more likely to form. ${ }^{34}$ Besides, the excessive production for fibrinogen, and coagulation factors V and VIII by the liver as compensation for hypoproteinemia might enlarge the coagulation pathway. ${ }^{35}$ Third, hypoalbuminemia increased the risk of stroke complications such as pneumonia, which significantly impaired survival status and functional recovery. ${ }^{36}$

In a recent subgroup analysis of the ALIAS trials, for patients with CE in the middle cerebral artery (NIHSS score $\geq 15$ ), there was a trend to be effective when highdose albumin therapy was started within 2 hours after onset, indicating that some specific subgroups might 
benefit from this treatment. ${ }^{37}$ Meanwhile, by careful fluid management and diuretic treatment, common complications included pulmonary oedema and heart failure after albumin infusion could be partially controlled. ${ }^{6}$ These remarkable findings indicated that a more precise and individualised application of albumin might be needed to re-examine the neuroprotection hypothesis. For patients with hypoproteinemia, positive therapy should be initiated as soon as possible. Also, the therapeutic options would be more effective by treating the underlying cause simultaneously, rather than mere albumin supplement. ${ }^{8}$ For patients with normal albumin, it might be more beneficial to take an appropriate dose of albumin infusion to specific subgroups of patients representative of preclinical settings while actively controlling complications.

Some prior studies have shown that high albumin level is associated with a better prognosis. However, it was based on comparison with low albumin level. ${ }^{10}$ Given the multiple physiological effects of albumin, there might be a ceiling effect in albumin therapy. More research is needed to find the sweet spot of serum albumin therapy. Furthermore, since albumin could pass through the blood-brain barrier, the strong adsorption function of albumin to transport relevant drugs to local damaged brain tissue will also have extraordinary effects on promoting functional recovery. ${ }^{38}$

This study has some limitations. First, only one period of serum albumin was measured, and there was no follow-up on the changes in albumin levels. Second, the serum albumin level was not tested immediately after the stroke. A previous study found that serum albumin levels might change during the first days after stroke, especially in patients with poor outcome. ${ }^{39}$ Therefore, our data may partially magnify the negative relationship between hypoalbuminemia and outcome. Third, serum albumin measurement of sub-centres was not uniform. In fact, the bromocresol green assay is a common method to measure albumin, but is affected by $\alpha 1$-globulin and $\alpha 2$-globulin, which might cause a positive bias, especially in severe hypoalbuminemia. Meanwhile, the bromocresol purple assay could avoid this effect. ${ }^{40}$ Therefore, our study may reduce the proportion of hypoalbuminemia, and weaken the correlation between low serum albumin level and poor prognosis. Fourth, there may be a selection bias when patients were enrolled. Some older patients with severe illness at the time of onset were more likely to refuse admission. However, they may be closely related to hypoalbuminemia. Fifth, despite no statistical bias, few studies were included in the meta-analysis, and the definition of poor functional outcome varied. More prospective studies are needed to validate the findings in the future.

\section{CONCLUSIONS}

In summary, low serum albumin level was a robust, independent predictor in poor functional outcome and mortality of patients with AIS or TIA. As a promising neuroprotective agent, serum albumin requires more further investigation.

\section{Twitter Yilong Wang @yilong}

Acknowledgements We thank the staff and participants of the CNSR-III studies for their contribution.

Contributors Y-JW had full access to all of the data in the study and takes responsibility for the integrity of the data and the accuracy of the data analysis. Y-JW designed the study. $\mathrm{HZ}, \mathrm{AW}$ and $\mathrm{XM}$ collected the data. $\mathrm{HZ}$ and $\mathrm{AW}$ wrote the manuscript. $\mathrm{HZ}, \mathrm{AW}$ and $\mathrm{YZ}$ completed the statistical analysis. JL, YJ, JJ, Y-LW, XZ and $\mathrm{HL}$ reviewed, edited and approved the final version.

Funding This work was supported by grant from National Key R\&D Program of China (2018YFC1312903), grant from the National Natural Science Foundation of China (81870905), grant from Beijing Municipal Science \& Technology Commission (Z181100001818001), grant from Beijing Municipal Administration of Hospitals Incubating Program (PX2020021) and grant from Beijing Excellent Talents Training Program (2018000021469G234).

Competing interests None declared.

Patient consent for publication Not required.

Provenance and peer review Not commissioned; externally peer reviewed. Data availability statement Anonymised data are available to other researchers on request for the sole purpose of replicating procedures or reproducing the results by directly contacting the corresponding author and their institutions.

Open access This is an open access article distributed in accordance with the Creative Commons Attribution Non Commercial (CC BY-NC 4.0) license, which permits others to distribute, remix, adapt, build upon this work non-commercially, and license their derivative works on different terms, provided the original work is properly cited, appropriate credit is given, any changes made indicated, and the use is non-commercial. See: http://creativecommons.org/licenses/by-nc/4.0/.

ORCID iDs

Hongyu Zhou http://orcid.org/0000-0002-9130-889X

Anxin Wang http://orcid.org/0000-0003-4351-2877

Hao Li http://orcid.org/0000-0002-8591-4105

Yongjun Wang http://orcid.org/0000-0002-9976-2341

\section{REFERENCES}

1 Boldt J. Use of albumin: an update. Br J Anaesth 2010;104:276-84.

2 Lang J, Katz R, Ix JH, et al. Association of serum albumin levels with kidney function decline and incident chronic kidney disease in elders. Nephrol Dial Transplant 2018;33:986-92.

3 Arques S. Human serum albumin in cardiovascular diseases. Eur J Intern Med 2018;52:8-12.

4 Belayev L, Liu Y, Zhao W, et al. Human albumin therapy of acute ischemic stroke: marked neuroprotective efficacy at moderate doses and with a broad therapeutic window. Stroke 2001;32:553-60.

5 Belayev L, Pinard E, Nallet $\mathrm{H}$, et al. Albumin therapy of transient focal cerebral ischemia: in vivo analysis of dynamic microvascular responses. Stroke 2002;33:1077-84.

6 Martin Renee' H, Yeatts SD, Hill MD. ALIAS (Albumin in Acute Ischemic Stroke) trials: analysis of the combined data from parts 1 and 2. Stroke 2016;47:2355-9.

7 Famakin B, Weiss P, Hertzberg V, et al. Hypoalbuminemia predicts acute stroke mortality: Paul Coverdell Georgia stroke registry. $J$ Stroke Cerebrovasc Dis 2010;19:17-22.

8 Seet RCS, Lim ECH, Chan BPL, et al. Serum albumin level as a predictor of ischemic stroke outcome. Stroke 2004;35:2435-6. author reply -6 .

9 Babu MS, Kaul S, Dadheech S, et al. Serum albumin levels in ischemic stroke and its subtypes: correlation with clinical outcome. Nutrition 2013:29:872-5.

10 Idicula TT, Waje-Andreassen U, Brogger J, et al. Serum albumin in ischemic stroke patients: the higher the better. The Bergen stroke study. Cerebrovasc Dis 2009;28:13-7.

11 Kimura Y, Ohji S, Ishiyama D, et al. Factors associated with functional recovery in Japanese patients with convalescent stroke stratified by age: a multicenter observational study. Int J Rehabil Res 2019;42:249-55.

12 Wang $Y$, Jing J, Meng X, et al. The third China national stroke registry (CNSR-III) for patients with acute ischaemic stroke or 
transient ischaemic attack: design, rationale and baseline patient characteristics. Stroke Vasc Neurol 2019;4:158-64.

13 Adams HP, Bendixen BH, Kappelle LJ, et al. Classification of subtype of acute ischemic stroke. definitions for use in a multicenter clinical trial. TOAST. trial of org 10172 in acute stroke treatment. Stroke 1993;24:35-41.

14 Samuels OB, Joseph GJ, Lynn MJ, et al. A standardized method for measuring intracranial arterial stenosis. AJNR Am J Neuroradiol 2000;21:643-6.

15 North American Symptomatic Carotid Endarterectomy Trial Collaborators, Barnett HJM, Taylor DW, et al. Beneficial effect of carotid endarterectomy in symptomatic patients with high-grade carotid stenosis. N Engl J Med 1991;325:445-53.

16 Inker LA, Astor BC, Fox CH, et al. KDOQI US commentary on the 2012 KDIGO clinical practice guideline for the evaluation and management of CKD. Am J Kidney Dis 2014;63:713-35.

17 Chen W, Pan Y, Zhao X, et al. Totaled health risks in vascular events score predicts clinical outcome and symptomatic intracranial hemorrhage in Chinese patients after thrombolysis. Stroke 2015;46:864-6.

18 Xu W-H, Dong C, Rundek T, et al. Serum albumin levels are associated with cardioembolic and cryptogenic ischemic strokes: Northern Manhattan study. Stroke 2014;45:973-8.

19 Toyoda K, Ninomiya T. Stroke and cerebrovascular diseases in patients with chronic kidney disease. Lancet Neurol 2014;13:823-33.

20 Liberati A, Altman DG, Tetzlaff $\mathrm{J}$, et al. The PRISMA statement for reporting systematic reviews and meta-analyses of studies that evaluate health care interventions: explanation and elaboration. J Clin Epidemiol 2009;62:e1-34.

21 Stroup DF, Berlin JA, Morton SC. Meta-analysis of observational studies in epidemiology: a proposal for reporting. Meta-analysis Of Observational Studies in Epidemiology (MOOSE) group. JAMA 2000;283:2008-12.

22 Ronit A, Kirkegaard-Klitbo DM, Dohlmann TL, et al. Plasma albumin and incident cardiovascular disease: results from the CGPS and an updated meta-analysis. Arterioscler Thromb Vasc Biol 2020;40:473-82.

23 Dziedzic T, Pera J, Slowik A, et al. Hypoalbuminemia in acute ischemic stroke patients: frequency and correlates. Eur J Clin Nutr 2007;61:1318-22.

24 Lyu YB, Zhou JH, Duan J, et al. [Association of plasma albumin and hypersensitive $\mathrm{C}$-reactive protein with 5 -year all-cause mortality among Chinese older adults aged 65 and older from 8 longevity areas in China]. Zhonghua Yu Fang Yi Xue Za Zhi 2019;53:590-6.
25 Liao L-Z, Zhang S-Z, Li W-D, et al. Serum albumin and atrial fibrillation: insights from epidemiological and Mendelian randomization studies. Eur J Epidemiol 2020;35:113-22.

26 Wang Y, Zhao X, Liu L, et al. Prevalence and outcomes of symptomatic intracranial large artery stenoses and occlusions in China: the Chinese intracranial atherosclerosis (CICAS) study. Stroke 2014;45:663-9.

27 Fanali G, di Masi A, Trezza V, et al. Human serum albumin: from bench to bedside. Mol Aspects Med 2012;33:209-90.

28 Gimbrone MA, García-Cardeña G. Endothelial cell dysfunction and the pathobiology of atherosclerosis. Circ Res 2016;118:620-36.

29 Geovanini GR, Libby P. Atherosclerosis and inflammation: overview and updates. Clin Sci (Lond) 2018;132:1243-52.

30 Gariballa SE, Parker SG, Taub N, et al. Influence of nutritional status on clinical outcome after acute stroke. Am J Clin Nutr 1998;68:275-81.

31 O'Neal WT, Claxton J'Neka S, Sandesara PB, et al. Provider specialty, anticoagulation, and stroke risk in patients with atrial fibrillation and cancer. J Am Coll Cardiol 2018;72:1913-22.

$32 \mathrm{He} \mathrm{H}$, Guo J. Serum albumin: a risk of stroke? Am J Emerg Med 2017;35:186-7.

33 Mikhailidis DP, Ganotakis ES. Plasma albumin and platelet function: relevance to atherogenesis and thrombosis. Platelets 1996;7:125-37.

34 Gyamlani G, Molnar MZ, Lu JL, et al. Association of serum albumin level and venous thromboembolic events in a large cohort of patients with nephrotic syndrome. Nephrol Dial Transplant 2017;32:157-64.

35 Singhal R, Brimble KS. Thromboembolic complications in the nephrotic syndrome: pathophysiology and clinical management. Thromb Res 2006;118:397-407.

36 Dziedzic T, Pera J, Klimkowicz A, et al. Serum albumin level and nosocomial pneumonia in stroke patients. Eur J Neurol 2006;13:299-301.

37 Khatri R, Afzal MR, Rodriguez GJ, et al. Albumin-induced neuroprotection in focal cerebral ischemia in the ALIAS trial: does severity, mechanism, and time of infusion matter? Neurocrit Care 2018;28:60-4.

38 Eady TN, Khoutorova L, Obenaus A, et al. Docosahexaenoic acid complexed to albumin provides neuroprotection after experimental stroke in aged rats. Neurobiol Dis 2014;62:1-7.

39 Makris K, Koniari K, Spanou L, et al. Prognostic significance of serum albumin level changes in acute ischemic stroke: the role of biological and analytical variation. Clin Chem Lab Med 2016;54:143-50.

40 Ueno T, Hirayama S, Sugihara M, et al. The bromocresol green assay, but not the modified bromocresol purple assay, overestimates the serum albumin concentration in nephrotic syndrome through reaction with $\alpha 2-$ macroglobulin. Ann Clin Biochem 2016;53:97-105. 